\title{
The Benefit of a Virtual 3D-Model for Patients Undergoing Patient Education for Planned Gynecological Operations Including Hysterectomy
}

\author{
Matthias Kiesel*1, Adam Kalisz ${ }^{2}$, Inga Beyers ${ }^{3}$, Achim Wöckel $^{1}$, Saskia-Laureen Herbert ${ }^{1}$, \\ Carolin Curtaz $^{1}$, Ralf Joukhadar ${ }^{1}$ and Christine Wulff ${ }^{1}$ \\ ${ }^{1}$ Department of Gynecology, University Hospital Würzburg, Germany \\ ${ }^{2}$ Department of Electrical, Electronic and Communication Engineering, Information Technology (LIKE), Germany \\ ${ }^{3}$ Institute of Electric Power Systems (IfES), Leibniz Universität Hannover, Germany \\ *Corresponding author: Matthias Kiesel, Department of Gynecology, University Hospital Würzburg, Germany
}

\section{ARTICLE INFO}

Received: 㠦 June 28, 2021

Published: 幽 July 07, 2021

Citation: Matthias Kiesel, Adam Kalisz, Inga Beyers, Achim Wöckel, Saskia-Laureen Herbert, et al., The Benefit of a Virtual 3D-Model for Patients Undergoing Patient Education for Planned Gynecological Operations Including Hysterectomy. Biomed J Sci \& Tech Res 37(1)-2021. BJSTR. MS.ID.005940.

Keywords: Virtual, 3D, Patient Education, Gynecology, Hysterectomy

Abbreviations: HE: Hysterectomy; SDM: Shared Decision Making; WLAN: Wireless Local Area Network; HSIL: High-Grade Squamous Intraepithelial Lesion; TLH: Total Laparoscopic Hysterectomies

\section{ABSTACT}

Introduction: Surgical interventions require profound preoperative patient education. In reality, the concerning physician faces various challenges, including great time pressure. We wanted to evaluate the impact of a self-made, virtual, simplified and interactive 3D-model of the female pelvis on the satisfaction and comprehension of patients undergoing patient education for planned hysterectomy.

Materials and Methods: With the open-source-program Blender we created a virtual model depicting the female pelvis. Patients of the control group (Group A, N = 26 ) received regular patient education. In the experimental group (Group $B, N=25$ ), we additionally used the virtual model. The patients rated the patient education with a questionnaire consisting of questions 1 - 5, with a numeric scale reaching from 1 ("very little" / "very poor") to 10 ("very much" / "very good"). Questions 6-10 offered open questions, which the patients answered using their own words. The answers were rated in right / wrong. We used Microsoft Excel for Mac, version 16.16.21, IBM SPSS Statistics version 24 and 25, Fisher's exact test and Mann-Whitney-U-Test.

Results: Group B rated the quality of the patient education higher in 5 of 5 questions with statistically significant differences in 4 of 5 questions. The questions 6-10 showed more correct answers in Group B for 5 of 5 questions with a trend in question 10 (p = $0,05)$. The other 4 questions showed no statistically significant differences.

Conclusion: A 3D-model of the female pelvis can improve patient education. Further research is needed.

\section{Introduction}

Surgical interventions need a sufficiently profound preoperative patient education, which normally requires written documentation. Concerning surgery such as hysterectomy (HE), its complexity and challenging anatomy has to be taken into account. Because of this, the preoperative preparation is crucial. One significant part of these procedures is a sufficiently thorough explanation for the patient. It should include the according medical indication, access path, anatomy, intraoperative procedures, chances of success, as well as possible complications, etc.. Especially in times of well-informed, self-determined patients and "Shared Decision Making" (SDM), a detailed, high quality patient education, tailored to the needs of each single patient, is pivotal. The literature also highlights the importance of SDM. Moreover, there are numerous demands for additional research as well as further improvements concerning 
SDM in clinical everyday life [1-9]. This involves elective surgeries for benign and malignant diseases. Furthermore, an "Informed Consent", which also is highly important from a legal point of view, requires a well-informed patient $[10,11]$. In reality, the physician leading such a conversation for preparing surgery faces organizational, structural and material challenges, including great time pressure. This makes it more difficult to meet the individual needs of each single patient.

According to our knowledge, the evaluation of the practical use of different anatomical models for explaining female pelvic surgery has only marginally been addressed until know. Two promising research projects, focusing on gynecological maladies, show rather few case numbers $(\mathrm{N}=1$ and $\mathrm{N}=5)[12,13]$. Baek, et al. used a model reassembling the real cervical carcinoma of the concerning patient. This model was used for the sake of illustration during the patient education [12]. Sayed Aluwee, et al. used replicas of real uteri of the concerning patients suffering from endometrial cancer. The individualized models were produced based on MRI- images and were also used during patient education [13]. Other research projects have up until now, mainly treated areas other than
Gynecology. There are concepts with real physical, as well as virtual models [14-16]. Many projects focus on the education of young physicians or medical students [17-20]. Schmitt, et al. and Wake, et al. could generate promising findings by using individualized, physical models made by 3D-printing for patient education of urological surgeries. The models were produced, based on clinical imaging $[21,22]$. Yet, the production of 3D-printed individualized models based on CT- or MRI-imaging, is complex and expensive. Consequently, such models seem only partly fitting for the regular clinical everyday work. This relatively small amount of data for possible improvements of patient education is surprising, as there have been interesting approaches concerning this issue, showing its potential [23].

In our work we wanted to evaluate the impact of a self-made, virtual, simplified, and interactive model of the female pelvis on the satisfaction and comprehension of patients undergoing patient education for a planned hysterectomy. Our hypothesis was that, by using the model, patients feel better informed and experience a measurable improvement of transferred knowledge, than patients undergoing patient education without the model.

\section{Materials and Methods}

\section{The 3D-Model}

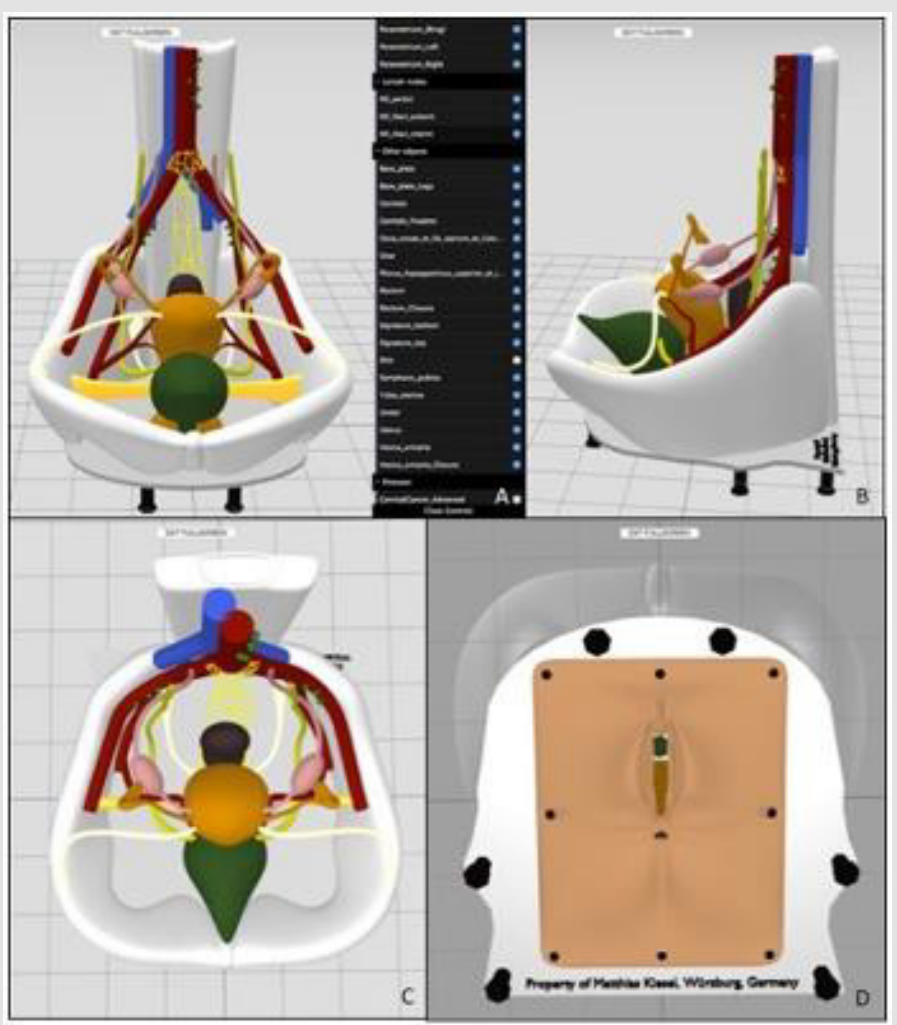

Figure 1: The virtual model.

A. Virtual model of the pelvis with drop-down-menu for showing and hiding of single parts.

B-D. Different views of the virtual model. Tilting the model to $90^{\circ}$ makes it possible to simulate the position of the female pelvis during gynecological examination. 
By using the open-source-program Blender, version 2.82, we created a virtual model depicting the female pelvis. It can be seen in Figure 1. The model incorporates the following anatomical structures: Skin of the abdomen and pelvis, navel, vulva, vagina, uterus with adnexae, pelvic bones, spine, pelvic floor with ligaments (round ligaments, sacro-uterine ligaments, parametrium), ureter, bladder, rectum, superior and inferior hypogastric plexus, exemplary arterial and venous vessels (aorta, internal and external iliac artery, uterine artery, inferior vena cava, iliac vein, internal iliac vein) as well as pelvic and para-aortic lymph nodes. The depicted structures, which the model contains, were selected according to clinical experience. The illustration of the anatomical structures was simplified and combined with the use of different colors, in order to ease the understanding for patients. All of the components of the model can be selected, shown and hidden in an individual sequence by using a menu on the right-hand side of the screen. In addition, one can zoom in and out as, well as move, rotate and tilt the model in all directions.

By doing so, an operation can be explained individually, step by step and questions posed by the patient can be answered immediately. The model can be shown after a short briefing, by using a regular computer or mobile device such as a tablet PC with a normal 2D-screen. Furthermore, the current view can be captured at any time by using a screenshot-function, thus enabling the physician to further explain certain steps of a procedure by adding personal notes or drawings directly on the screen. These pictures can also be printed and added to the written patienteducation document. The model is shown by using a passwordprotected web-based application (Webapp). This makes it possible to use the model from every computer or mobile device, which can enter the internet. Access is also possible by using the hospital's Wireless Local Area Network (WLAN), enabling hospital staff to work with the model from different rooms at the same time. In our project, we presented the model to our patients by using a tablet-PC (Apple iPad Pro, 12,9 inch, model A 1895) with the browser Google Chrome.

\section{Data Recruitment}

From April 2019 until March 2020, all in all 51 patients were prepared for surgery including hysterectomy by the same physician at the department of Obstetrics and Gynecology of the University Clinic of Würzburg (single-center-study). The patients of the control group (Group A) received regular preoperative patient education. The official, standardized patient-education sheets provided by the company Thieme were used. They depict single steps of procedures and anatomical structures of the female pelvis via 2D-pictures. In the experimental group (Group B) we additionally used the described virtual model for the patient education. The patients were randomly assigned to the two groups A or B by picking a ticket from a box (balanced randomization). Group A included 26 and Group B 25 patients. The patients' age ranged from 24 to 82 years with a median age of 49 years. Table 1 shows the type and number of different preoperative diagnoses, which led to the indication for surgery and concerning patient education. Oncologic diseases (51\% of cases) consisted of 8 cases of cervical cancer, 6 cases of endometrial cancer and 5 cases of ovarian cancer, as well as one case of rectal cancer infiltrating the uterus.

Table 1: Type and number of different preoperative diagnoses.

\begin{tabular}{|c|c|}
\hline Diagnosis & Number \\
\hline Cervical carcinoma & 8 \\
\hline Endometrial cancer & 6 \\
\hline Ovarian cancer & 5 \\
\hline $\begin{array}{c}\text { Rectal cancer infiltrating the uterus } \\
\text { High-grade squamous intrapithelial lesion (HSIL) of the } \\
\text { Myoma }\end{array}$ & 1 \\
\hline Dys- and hypermenorrhoea & 22 \\
\hline
\end{tabular}

Additionally, there were 6 cases of high-grade squamous intraepithelial lesion (HSIL) of the cervix. Benign diseases (49 $\%$ of cases) consisted of 22 cases of symptomatic myoma and 3 cases of idiopathic dys- and hypermenorrhea. All surgeries, which were prepared, contained hysterectomy. 23 abdominal hysterectomies, 25 total laparoscopic hysterectomies (TLH) and 3 vaginal hysterectomies were performed. All patient educations for both groups were performed by one single physician. The duration of each conversation was at least 30 minutes. At the end of the conversation, the patients rated the quality of the patient education by using a questionnaire, which we created ourselves. Consequently, there is no external validation. By using a self-made questionnaire, we aimed to assess certain targeted aspects of the virtual model. The questionnaire consists of a first part with the questions 1 to 5 , which are to be answered by choosing from a scale of 1 ("very little" or "very poor") to 10 ("very much" or "very good"). They are shown in Table 2. In this manner, we aimed to measure the patients' personal, subjective opinion of the model.

Table 2: Questions 1 to 5, which are to be answered by choosing from a scale reaching from 1 ("very little" or "very poor") to 10 ("very much" or "very good").

1. How well informed do you feel about the planned operation?

2. Did you understand the single steps of the operation?

3. Did you understand the anatomical structures and relationships?

4. How insecure do you feel about the planned operation?

5. How comprehensible was the education? 
Questions 1 to 10 are similar between Group A and Group B. The second part of the questionnaire consists of questions 6 to 10 and offers open questions to the patients, which they answer by using their own words and formulations. This was supposed to objectively assess how much the patients had really understood and remembered from the patient education. Questions 6 to 10 are shown in Table 3, together with notes to the assessment of the answers. The questionnaires of the patients of Group B, who received the 3D-model of the female pelvis for patient education, additionally contained targeted questions towards the 3D-model. These questions were answered by also choosing from a scale of 1 ("very little" or "very poor") to 10 ("very much" or "very good"). They can be seen in Table 4.

Table 3: Open questions to be answered by the patient's from Group A and B using their own words and formulations.

6. For which reason(s) will the planned operation be performed? (This question was rated as correctly answered, if the patient stated the diagnosis correctly. We accepted regular German terms (as well as Latin technical terms). Mistakes in spelling were accepted.)

7. Which organs / structures will be removed during the planned operation? (For answering this question, the patients had to name varying amounts of structures, depending on the kind of planned procedure. Stating the uterus as organ to be removed was obligatory. If the diagnosis was a benign disease, the fallopian tubes also had to be named in order to correctly answer the question. In case of more complex surgery, e.g. treatment of ovarian cancer, the question was rated as correctly answered, if the patient stated at least three correct structures including the uterus. The answer was immediately rated as wrong, if one incorrect structure was named.).

8. Through which access path will your uterus be removed? (This question aimed at the differences between laparotomic, laparoscopic and vaginal hysterectomy. If a patient undergoing TLH without morcellement wrote that her vagina was the access path, through which the uterus was removed, the question was rated as correct.).

9. Which risks does the planned operation have? (The patient's answer was seen as correct, if at least three different correct risks were stated.).

10. Please name or describe neighboring organs / structures close to the uterus. (In our eyes, patients correctly answered this question, if they enumerated at least three correct pelvic structures / organs.).

Table 4: Questions for the patients of Group B, rating the $3 \mathrm{D}$-model. These questions were to be answered by choosing from a scale reaching from 1 ("very little" or "very poor") to 10 ("very much" or "very good").

11. How helpful was the 3D-model for your understanding of the anatomy and the planned operation?

12. Would you recommend the use of 3D-models for patient education?

13. Would you approve of a real 3D-model, e.g. made out of plastic, for patient education?

14. Would you approve of an individualized model, showing your personal medical condition, which shall be treated?

15. Was the interactive step-by-step presentation with the 3D-model helpful for your understanding of the planned procedure and the concerning anatomy?

\section{Ethical Standards}

The participation in this study was voluntary. All patients signed a declaration of consent, that the data of the questionnaires was allowed to be collected for scientific purposes. After the patient education and subsequent completion of their questionnaire, patients of Group A were allowed to see the 3D-model as well, if they wished to do so, in order to prevent any disadvantage for them. Patients were allowed to bring along relatives for the patient education, if they wanted to. A certificate of non-objection was obtained from the Ethics Committee for the University of Würzburg from 11.04.2019 (application number: 2019030701). The model is privately owned by the corresponding author with copyright claims.

\section{Statistics}

For evaluation of the data we used Microsoft Excel for Mac, version 16.16.21 and IBM SPSS Statistics version 24 and 25. The Mann-Whitney-U-Test was used for testing the metric variables (choosing on a scale from 1 to 10 points) of questions 1 to 5 between Group A and B for significance. Fisher's exact test was used for testing the answers to the dichotomous questions 6 to 10 (right or wrong) between Group A and B for significance. The two named tests were used, as there was no normal distribution of the data. The level of significance was set at $5 \%$. The symbol * indicates significant differences.

\section{Results}

\section{Subjective Rating of the Patient Education}

Patients assigned to Group B, who received patient education together with the described 3D-model of the female pelvis, rated the quality of the patient education higher than the patients from Group A, who received a regular patient education without the model. The results are summarized in Figure 2, which displays the differences in the median answers to the questions 1 to 5 between Group A and B. These questions refer to the feeling of quality of the patient education (question 1), the subjective understanding of the steps of the surgery (question 2) and the anatomical relations (question 3), the patient's insecurity (question 4) and the perceived comprehensibility for the patient education (question 5). For these parameters, we found that patients from Group B rated the quality of the patient education higher than those from Group A. Except for question $4(p=0,286)$, the differences in question $1(p=0,024), 2(p$ $=0,004), 3(\mathrm{p}<0,001)$ and $5(\mathrm{p}=0,007)$ are statistically significant.

\section{Objectively Transferred Knowledge}

The questions 6-10 aim especially at the patient's understanding of the planned operation and the corresponding anatomy. Results are summarized in Figure 3. All questions in section 6-10 were answered correctly more often by Group B. These differences were not statistically significant (question 6: $\mathrm{p}=0,67$, question $7: \mathrm{p}=$ 1,0, question 8: $p=0,11$, question 9: $p=1,0$, question $10 p=0,05$ ). Yet, concerning question 10 (Please name or describe neighboring organs / structures close to the uterus.) a tendency can be seen with $\mathrm{p}=0,05$. 


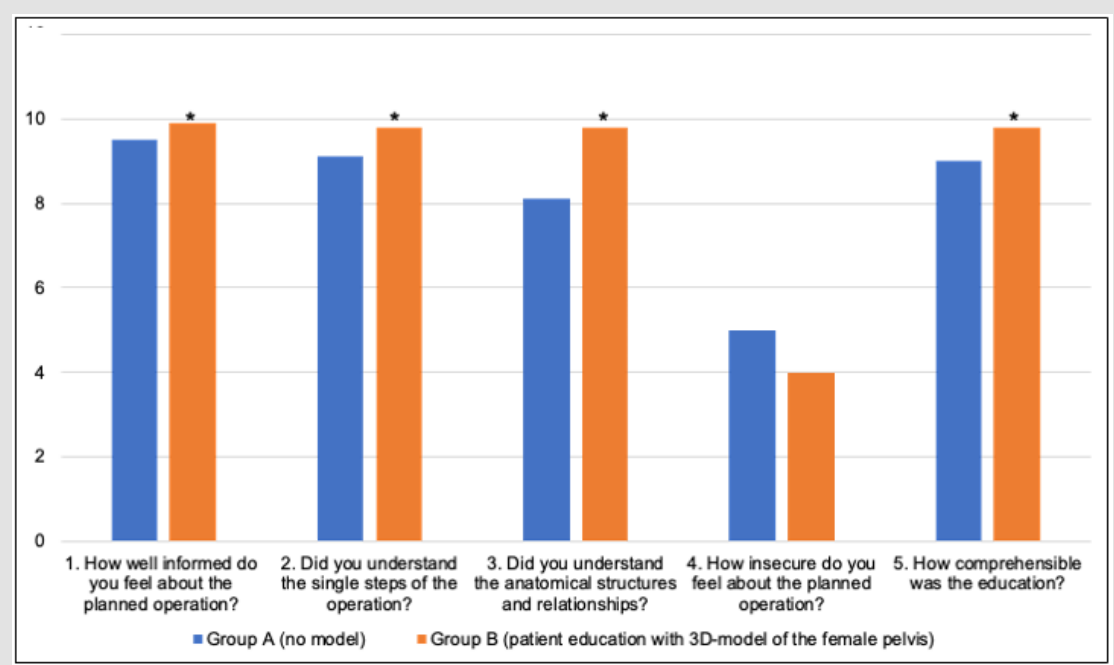

Figure 2: Subjective assessment of the patient education.

Depiction of the assessment of the patient education, comparing Group A ( $\mathrm{N}=26$, no 3D-model) with Group B ( $\mathrm{N}=25$, 3D-model of the female pelvis for patient education).

The questions 1 to 5 are to be answered by choosing from a scale reaching from 1 ("very little" or "very poor") to 10 ("very much" or "very good"). The level of significance was set at $5 \%$. The symbol * indicates significant differences. Mann-WhitneyU-Test.

1) Average of the points for question 1: Group A: 9,46, Group B: 9,88, $\left(p=0,024^{*}\right)$

2) Average of the points for question 2: Group A: 9,12, Group B: 9,80, $\left(p=0,004^{*}\right)$

3) Average of the points for question 3: Group A: 8,08, Group B: 9,80, $\left(p<0,001^{*}\right)$

4) Average of the points for question 4: Group A: 4,96, Group B: 4,04, $(p=0,286)$

5) Average of the points for question 5: Group A: 9,04, Group B: 9,84, $\left(p=0,007^{*}\right)$.

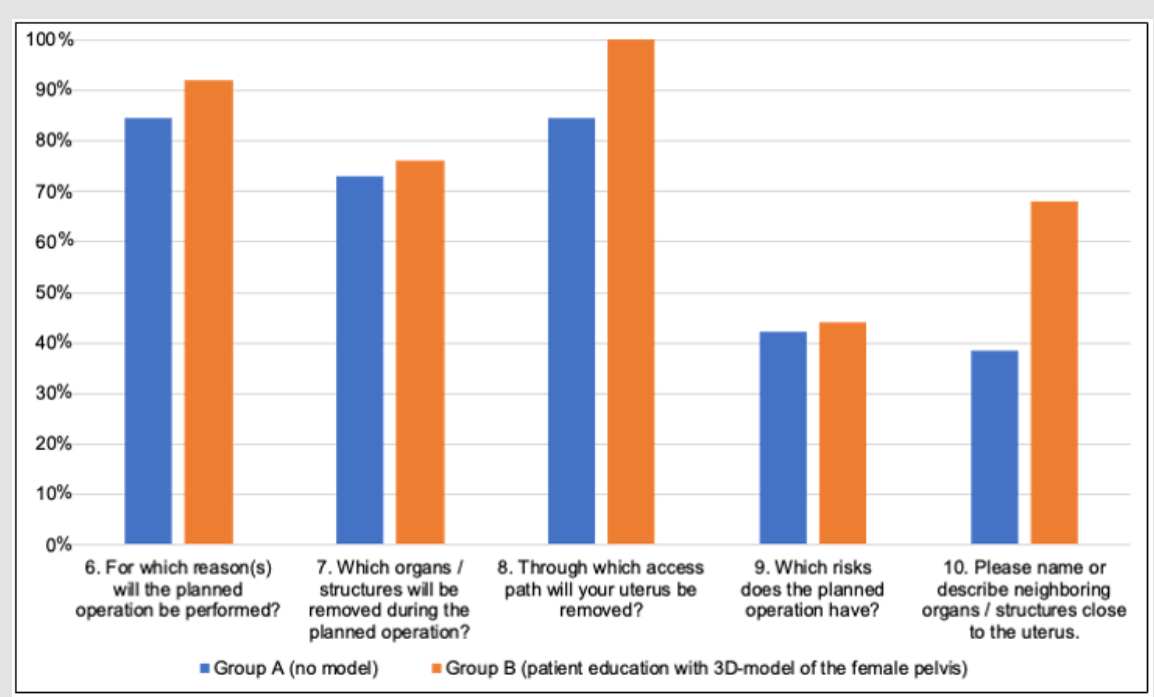

Figure 3: Correctly answered questions to the patient education Percentage of the correctly answered questions 6 to 10. Group A $(\mathrm{N}=26)$, Group B $(\mathrm{N}=25)$.

These open questions were answered by the patients using their own wording. The answers were then assessed as right or wrong (dichotomous questions). Fisher's exact test was used.

6) Percentage of correct answers for question 6: Group A: 84,6\%, Group B: 92,0\%, (p=0,67).

7) Percentage of correct answers for question 7: Group A: 73,1\%, Group B: 76,0\%, $(p=1,0)$.

8) Percentage of correct answers for question 8: Group A: 84,6\%, Group B: 100,0\%, (p=0,11).

9) Percentage of correct answers for question 9: Group A: 42,3\%, Group B: 44,0\%, $(p=1,0)$.

10) Percentage of correct answers for question 10: Group A: 38,5\%, Group B: 68,0\%, $(p=0,05)$. 


\section{Specific Rating of the 3D-Model}

Finally, the patients' evaluation of the 3D-model by Group B was analyzed (Figure 4). The average rating of question 11 (How helpful was the 3D model for your understanding of the anatomy and the planned operation?) was 9,9 of 10 possible points. On average, the patients from Group B rated question 12 (Would you recommend the use of 3D-models for patient education?) with 9,6 points. In addition, question 13 (Would you approve of a real 3D-model, e.g. made out of plastic, for patient education?) was answered with an average of 5,8 points. Moreover, patients rated question 14 (Would you approve of an individualized model, showing your personal medical condition, which shall be treated?) with an average of 8,1 points. Furthermore, patients rated question number 15 (Was the interactive step-by-step presentation with the 3D-model helpful for your understanding of the planned procedure and the concerning anatomy?) with 9,9 points.

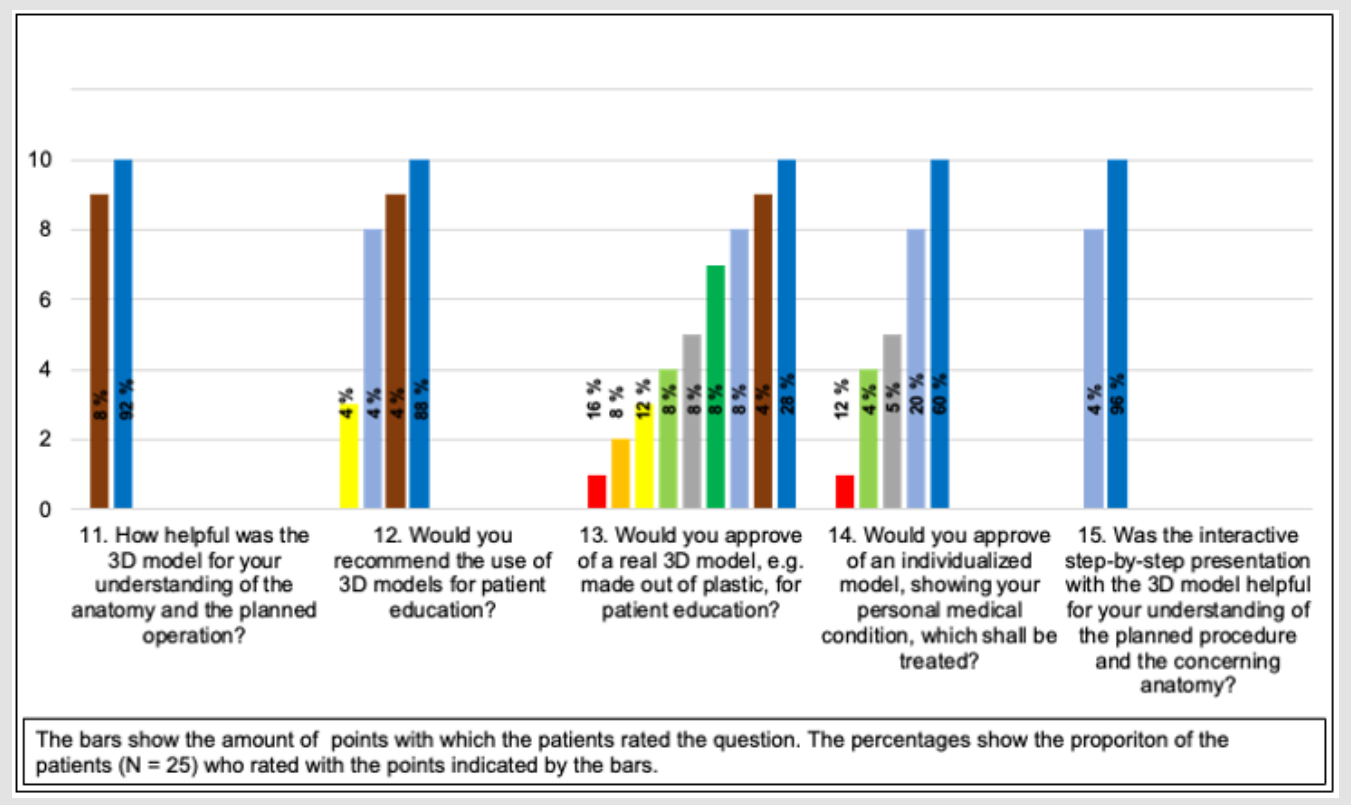

Figure 4: Evaluation of the 3D-model.

Percentages of the patient's evaluations of the 3D-model by Group B $(\mathrm{N}=25)$. The possible rating ranges from 1 ("very little" or "very poor") to 10 ("very much" or "very good).

\section{Discussion}

The results of this study show the successful use of a virtual, simplified interactive model for patient education of women undergoing pelvic surgery including hysterectomy. According to our knowledge, this is the first time such a study has been conducted for gynecological patients. The successful use of the model is indicated by questions 1 to 5 . They all show that patients from group B gave better ratings for the patient education than patients from Group A. In addition, questions 6 to 10 , which were to be answered by the patients using their own words, revealed more correct answers in Group B than in Group A. Our findings, that patients approve of a visualization of the concerning anatomy during patient education, equals the findings from Baek, et al. und Sayed Aluwee, et al.. However, the named authors used individualized, physical models. Additionally, these studies show relatively small case numbers ( $\mathrm{N}=5$ and $\mathrm{N}=1$ ), which can be explained with the resourceintensive production of such "hardware"-models $[12,13]$. Schmitt, et al. present larger case numbers with $\mathrm{N}=25$ in both control- and experimental group, but the data was generated for urological diseases and procedures, again using 3D- printed, individualized models [22]. The results from Schmitt, et al. nevertheless align with our findings and indicate that visualization is beneficial for patients during patient education.

Questions 1 to 5 display that patients in Group B feel better educated than patients in Group A, who had no 3D-model for their patient education. These subjectively answered questions focused on the feeling of quality of the patient education (question 1), the subjective understanding of the steps of the surgery (question 2) and the anatomical relations (question 3), the patient's insecurity (question 4), as well as the comprehensibility for the patient education (question 5). These differences between Group A and B were statistically significant for questions 1, 2, 3 and 5. Concerning question 4 (How insecure do you feel about the planned operation?) the difference to Group A was not statistically significant. This could show, that the explaining physician emphasized the possible risks of the concerning procedure equally thorough in both groups, not biasing on purpose. The descriptive differences between Group A and B for questions 1 to 5 were mostly little. This could again be an 
indication that both groups were informed well during the patient education and that a deliberate bias from the explaining physician is unlikely. In addition, this could also highlight the importance of a thorough and sensitive conversation itself for patient education, making the chosen method (pen and paper versus 3D-model) non-crucial. Nevertheless, our findings indicate that the use of a 3D-model can improve the patient's comprehension and satisfaction.

On average, Group B gave more correct answers to each of the single questions in question group 6-10, than Group A. With these questions we wanted to determine if there was an objectively improved patient education in Group B, since they asked the patients to actively reproduce the information. From a descriptive point of view these differences were considerable, raging up 29,5 $\%$ in question 10 ("Please name or describe neighboring organs / structures close to the uterus."). Yet, the differences in questions 6-10 between Group A and B were not statistically significant. Nevertheless, with $\mathrm{p}=0,05$ for question 10 , a tendency can be seen, favoring the 3D-model. Thus, taking into account the small case number of 26 patients for Group A and 25 patients for Group $B$, further research with a greater amount of patients is needed, in order to receive more clear findings whether an objectively demonstrable increase of transferred knowledge is possible via the described virtual model.

The patients in Group B approved of the virtual model (see questions 11, 12 and 15). Additionally, they expressed interest into further development such as an individualized model illustrating certain diseases (see question 14). This need should be met in future studies. Question 13 (Would you approve of a real 3D-model, e.g. made out of plastic, for patient education?) with an average rating of 5,8 on a scale from 1 to 10 , shows a certain skepticism from some patients and poses in interesting contrast to the stated data above. Looking at the results more closely, one can see that on the one hand $36 \%$ rated this question with 1 to 3 points, showing disapproval. On the other hand $40 \%$ of the patients would like such a physical model, as they rated with 7 to 10 points. Combining the findings of questions 13 and 14, we expect a real, physical model of the female pelvis, being able to show different gynecologic diseases to be promising for patient educations. This should be further evaluated in future research projects. A possible factor of bias in our study could be the heterogenity of the patient-group, as well as the types of surgery. The variation reached from abdominal to laparoscopic and vaginal hysterectomy, due to benign, as well as oncological diseases. We must assume that patients with a malignant disease suffered from greater anxiety and possibly less concentration during patient education than patients with benign diseases. The small case number with $\mathrm{N}=26$ for Group $\mathrm{A}$ and $\mathrm{N}=$ 25 for Group B must be seen as a relativizing factor of our study.
Lastly, a subjectively well informed patient is more likely to be satisfied with the ongoing procedures she experiences during her stay at the hospital, especially as the patient education often is one of the "first impressions" the patient receives from the hospital and its team. In addition to the demand for Shared Decision Making and Informed Consent, this should also be taken into account from a legal point of view.

\section{Conclusion}

Our data shows that a self-made, simplified, virtual and interactive 3D-model of the female pelvis can be of help for patient education, confirming our hypothesis. Further research, including larger case numbers, also as multi-center-studies, ought to be added. In addition to evaluating the patients' opinion directly after the patient education with the model, it would be of interest to evaluate the patients' overall satisfaction with the experienced treatment after having left the hospital. Adding to this, it should be evaluated how physicians using the model for their patient educations, rate the model themselves from a medical user point of view. Moreover, the patients' claim for an individualized virtual model should be met, as well as further evaluating the patients' skepticism concerning a real 3D-model, e.g. made out of plastic. We expect a real, physical model of the female pelvis, being able to show different gynecologic diseases, to be promising for patient educations. This should be further evaluated in future research projects.

\section{Conflicts of Interest}

The authors declare that they have no competing interests

\section{Acknowledgment}

Special thanks goes to PD Dr. med. Dr. med. dent. Klaus-Kristian Würzler for his help concerning IT-support.

\section{References}

1. de Mik SML, FE Stubenrouch, R Balm, DT Ubbink (2018) Systematic review of shared decision-making in surgery. Br J Surg 105(13): 17211730.

2. Ubbink DT, MG Hageman, DA Legemate (2015) Shared Decision-Making in Surgery. Surg Technol Int 26: 31-36.

3. Hester N, CL Pang, A Cho, R Kasivisvanathan, M Gooneratne (2019) Shared perioperative decision making: a shift in the doctor-patient paradigm. Br J Hosp Med (Lond) 80(4): 216-219.

4. De Oliveira GS, M Errea, J Bialek, MC Kendall, RJ McCarthy (2018) The impact of health literacy on shared decision making before elective surgery: a propensity matched case control analysis. BMC Health Serv Res 18(1): 958.

5. Page AE (2015) Safety in surgery: the role of shared decision-making. Patient Saf Surg 9: 24.

6. Ogburn T (2014) Shared decision making and Informed Consent for hysterectomy. Clin Obstet Gynecol 57(1): 3-13. 
7. Jones JW, LB McCullough, BW Richman (2005) Informed Consent: it's not just signing a form. Thorac Surg Clin 15(4): 451-460, v.

8. Pallett AC, BT Nguyen, NM Klein, N Phippen, CR Miller, et al. (2018) A randomized controlled trial to determine whether a video presentation improves Informed Consent for hysterectomy. Am J Obstet Gynecol, 2018. 219(3): 277.e1-277.e7.

9. Glaser J, S Nouri, A Fernandez, RL Sudore, D Schillinger, et al. (2020) Interventions to Improve Patient Comprehension in Informed Consent for Medical and Surgical Procedures: An Updated Systematic Review. Med Decis Making 40(2): 119-143.

10. Hanson M, D Pitt (2017) Informed Consent for surgery: risk discussion and documentation. Can J Surg 60(1): 69-70.

11. Godinho AM, LH Lanziotti, BS de Morais (2010) Informed Consent: the understanding of lawyers and courts. Rev Bras Anestesiol 60(2): 207214, 119-123.

12. Baek MH, DY Kim, N Kim, CC Rhim, JH Kim, et al. (2016) Incorporating a 3-dimensional printer into the management of early-stage cervical cancer. J Surg Oncol 114(2): 150-152.

13. Sayed Aluwee S, X Zhou, H Kato, H Makino, C Muramatsu, et al. (2017) Evaluation of pre-surgical models for uterine surgery by use of threedimensional printing and mold casting. Radiol Phys Technol 10(3): 279285.

14. Balaya V, JF Uhl, A Lanore, C Salachas, T Samoyeau, et al. (2016) 3D-modeling of the female pelvis by Computer-Assisted Anatomical Dissection: Applications and perspectives. J Gynecol Obstet Biol Reprod (Paris) 45(5): 467-477.

15. Barbosa MZ, DS Zylbersztejn, LA de Mattos, LF Carvalho (2019) Threedimensional printing models on reproductive surgery and human reproduction: systematic review and critical applications. Minerva Ginecol 71(3): 235-244.

16. Jones DB, R Sung, C Weinberg, T Korelitz, R Andrews (2016) ThreeDimensional Modeling May Improve Surgical Education and Clinical Practice. Surg Innov 23(2): 189-195.

17. Pujol S, M Baldwin, J Nassiri, R Kikinis, K Shaffer (2016) Using 3D-modeling Techniques to Enhance Teaching of Difficult Anatomical Concepts. Acad Radiol 23(4): 507-516.

18. Marconi S, L Pugliese, M Botti, A Peri, E Cavazzi, et al. (2017) Value of 3D printing for the comprehension of surgical anatomy. Surg Endosc 31(10): 4102-4110.

19. Smith CF, N Tollemache, D Covill, M Johnston (2018) Take away body parts! An investigation into the use of 3D-printed anatomical models in undergraduate anatomy education. Anat Sci Educ 11(1): 44-53.

20. Wu AM, K Wang, JS Wang, CH Chen, XD Yang, et al. (2018) The addition of 3D printed models to enhance the teaching and learning of bone spatial anatomy and fractures for undergraduate students: a randomized controlled study. Ann Transl Med 6(20): 403.

21. Wake N, AB Rosenkrantz, R Huang, KU Park, JS Wysock, et al. (2019) Patient-specific 3D printed and augmented reality kidney and prostate cancer models: impact on patient education. 3D Print Med 5(4): 1-8.

22. Schmit C, J Matsumoto, K Yost, A Alexander, L Ness, et al. (2019) Impact of a 3D printed model on patients' understanding of renal cryoablation: a prospective pilot study. Abdom Radiol (NY) 44(1): 304-309.

23. Holubar SD, JP Hassinger, EJ Dozois, JC Camp, DR Farley, et al. (2009) Virtual pelvic anatomy and surgery simulator: an innovative tool for teaching pelvic surgical anatomy. Stud Health Technol Inform 142: 122124.

$\begin{array}{ll}\text { BIOMEDICAL } & \text { Assets of Publishing with us } \\ \text { RESEARCHES } & \text { Global archiving of articles } \\ \text { - Immediate, unrestricted online access }\end{array}$

